\title{
Affective Touch at a Distance
}

\author{
Gijs Huisman \\ Human Media Interaction Group \\ University of Twente \\ Email: gijs.huisman@utwente.nl
}

\author{
Aduén Darriba Frederiks \\ Digital Life Centre \\ Amsterdam University of Applied Sciences \\ Email: a.darriba.frederiks@hva.nl
}

\author{
Dirk Heylen \\ Human Media Interaction Group \\ University of Twente \\ Email: d.k.j.heylen@utwente.nl
}

\begin{abstract}
Touch is an important modality for affective communication between individuals. Here we present a system, named the TaSST (Tactile Sleeve for Social Touch), that allows two people to communicate different types of touch at a distance by touching their own forearm. In this paper we will introduce the TaSST and present a demo setup using two TaSST sleeves.
\end{abstract}

\section{INTRODUCTION}

In interactions between people, touch is important in the forming of affiliative behavior and the maintenance of social bonds [1]. Touch can increase compliance to requests [2], can have stress reducing effects [3], is used to communicate affective states, and can even serve as a channel for communicating discrete emotions [4]. In summary, touch as a communication channel between people has a strong inherit affective connotation. This can be intuitively understood when thinking of being touched by a loved one as compared to being touched accidentally by a stranger on a busy train. The former is a highly positive affective experience, while the latter will most likely elicit negative affect. Effects of touch, similar to the ones described above, are being studied in computer mediated communication as well. Using haptic feedback technology, touches can be communicated at a distance. The effect of touch on compliance to requests has been found to be of similar size for touch mediated by technology, compared to real touch [5]. Mediated touch has been found to elicit emotional responses during a story telling scenario [6], and can be used to communicate discrete emotions [7]. Additionally, a host of devices that serve to explore touch at a distance, have been constructed (see [8] for an overview). Where most of these devices only communicate a single type of touch (e.g. hug, poke), our aim was to provide users with more freedom of expression in their tactile communication. For this reason we developed the TaSST (Tactile Sleeve for Social Touch)[9].

\section{THE TASST}

The TaSST is a wearable, smart-textile sleeve, that is worn on the forearm. The sleeve consists of a vibration motor output layer, and a conductive wool force-sensitive input layer. When two people both wear a TaSST, a touch applied to the input layer of one sleeve is communicated to the output layer of the other sleeve. The amount of force applied to the input layer of one sleeve controls the intensity of vibration of the vibration motors in the output layer of the other sleeve.

\section{A. TaSST design iterations}

The TaSST was designed to be worn on the forearm because the forearm is relatively sensitive to vibrotactile stimulation [10] and is an appropriate location for social touch to
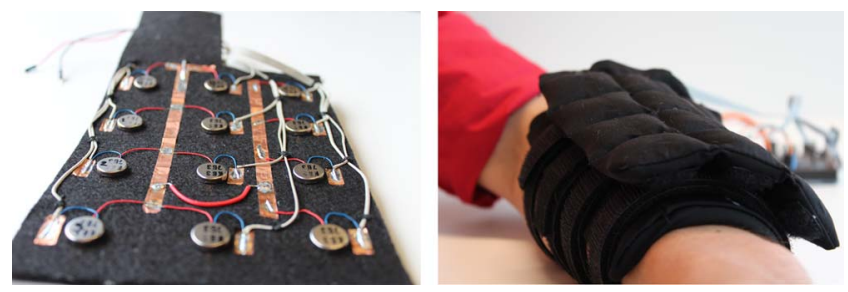

Fig. 1. The first version of the TaSST. The output layer on the left, and the input layer attached to the top of the output layer on the right

occur [4]. Because the TaSST was designed to be a wearable interface, we opted for a textile-based design that would be relatively comfortable to wear on the body. In the first version of the TaSST the input layer consisted of a 4 by 3 grid of $40 \mathrm{~mm}$ by $40 \mathrm{~mm}$ Lycra pads, filled with conductive wool (Bekeart Bekinox w12/18). By measuring changes in the internal resistance of the wool, touches of different intensities could be detected. The output layer of the first TaSST consisted of a 4 by 3 grid of pancake style eccentric mass vibration motors (KOTL C1226A001F) attached horizontally to a felt sheet (Figure 1)[9].

Using this first version of the TaSST we conducted a study in which participants received a number of different touches that were prerecorded by the experimenters using the input layer [9]. Participants received variations of simple, protracted, and dynamic touches [1] through the output layer and were asked to imitate the touch they thought they received. Overall we found that participants were most successful in imitating protracted, simple, and dynamic touches respectively.

Based on this first study a number of changes were made to the original design of the TaSST. The TaSST 1.5 [9] used felted conductive wool pads for the input layer, had excess material removed from the output layer, and had the vibration motors placed perpendicular to the surface of the output layer (Figure 2). The TaSST 1.5 was used in a study into the expression of

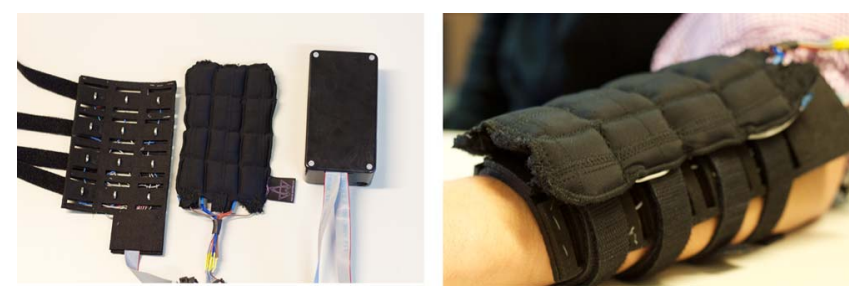

Fig. 2. The TaSST 1.5. The output layer, input layer and control box on the left, and the TaSST attached to the forearm on the right. 


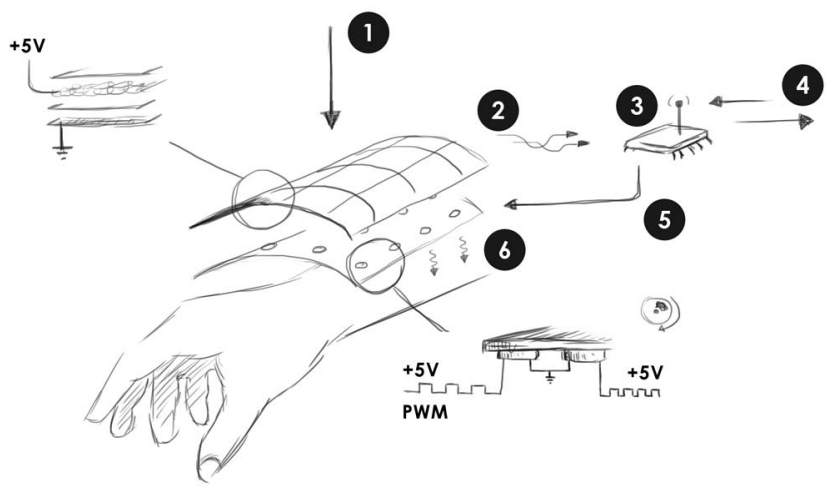

Fig. 3. Schematic of the TaSST 2. The input layer consists of layers of knitted conductive wool pads and isolation pads. The output layer is controlled using Pulse Width Modulation (PWM). Force is applied to the input layer (1), data is transfered (2) through an Zigbee wireless module (3) to a second sleeve (4, 5 ), where the output layer (6) is activated.

discrete emotions using the sleeve [11]. Participants wore the TaSST 1.5 around their non-dominant arm and were presented with eight different emotion words taken from [4]. Participants were asked to express these emotions using the input layer of the sleeve, record these expressions using a purpose-built computer program, and then play back the expressions to themselves. Participants could repeat this procedure as often as they liked. In addition, participants were asked to describe how they attempted to express each emotion. We found some indications for specific patterns in the expression of emotions using the TaSST, though results were inconclusive.

\section{DEMO OUTLINE}

For the demo a new version of the TaSST, TaSST 2.0, will be used (Figure 3). This new version of the TaSST features the new input layer which uses knitted wool pads (Figure 4) that combine capacitive and piezo-resistive sensing, resulting in a more stable and accurate input layer. The demo setup will consist of two sleeves which will communicate directly, and wirelessly. Touches applied to the input layer of one sleeve will be directly felt as a vibration on the other sleeve (Figure 3). This is the first time two TaSSTs will be used in this manner for direct, bi-directional communication. Visitors of the demo booth will be separated by an opaque screen. A monitor running a video calling application (e.g. Skype) will also be present. We will first allow visitors to explore using the sleeve with and without visual feedback of the other visitor. The aim is to provide visitors with a basic sense of being touched at a distance by another person, and get an idea of the influence of visual feedback of the other person on the communication through touch at a distance. We will encourage visitors to explore the communication of different types of touch, such as stroking, poking, and squeezing [9]. Next, we will present one visitor with a random emotion word and ask this visitor to express this emotion to the other visitor using the TaSST. Here we hope to build on results from our earlier study into the expression of emotions [11], and see if visitors, using the new input layer of the TaSST 2.0, can successfully communicate different affective states through remote touch. This way we hope to introduce participants to mediated touch as a novel affective communication modality.
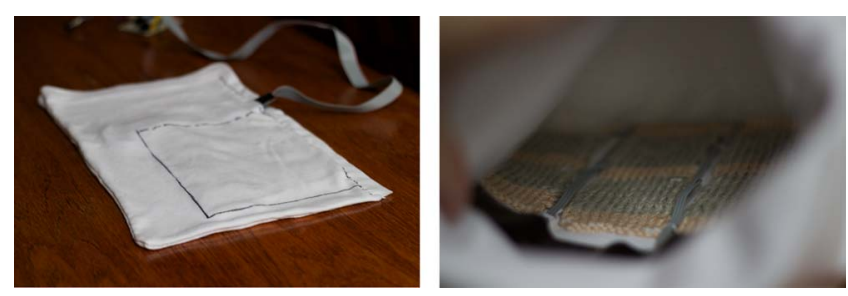

Fig. 4. A prototype of the TaSST 2.0 input layer. The left image shows the input layer covered with fabric. The right image shows the inside of the input layer. On the right the knitted wool sensor pads are visible.

\section{CONCLUSION}

In this paper we introduced the TaSST, and presented different design iterations of the sleeve based on user studies. We outlined the setup of a demo using two TaSST sleeves. The aim of the demo is to introduce affective touch mediated through technology as a novel channel of affective communication. Such a channel could potentially be relevant in remote communication (e.g. Skype), and interaction in virtual environments (e.g. communication with virtual agents).

\section{ACKNOWLEDGMENT}

This publication was supported by the Dutch national program COMMIT.

\section{REFERENCES}

[1] I. Morrison, L. Löken, and H. Olausson, "The skin as a social organ," Experimental Brain Research, vol. 204, pp. 305-314, 2010.

[2] N. Guéguen and C. Jacob, "The effect of touch on tipping: an evaluation in a french bar," International Journal of Hospitality Management, vol. 24, no. 2, pp. $295-299,2005$.

[3] T. Field, "Touch for socioemotional and physical well-being: A review," Developmental Review, vol. 30, no. 4, pp. 367-383, 2010.

[4] M. J. Hertenstein, R. Holmes, M. McCullough, and D. Keltner, "The communication of emotion via touch." Emotion, vol. 9, no. 4, pp. 566573, 2009.

[5] A. Haans and W. A. IJsselsteijn, "The Virtual Midas Touch: Helping Behavior After a Mediated Social Touch," IEEE Transactions on Haptics, vol. 2, no. 3, pp. 136-140, 2009.

[6] R. Wang, F. Quek, D. Tatar, K. S. Teh, and A. Cheok, "Keep in touch: channel, expectation and experience," in Proceedings of CHI '12. ACM, 2012, pp. 139-148.

[7] J. Bailenson, N. Yee, S. Brave, D. Merget, and D. Koslow, "Virtual interpersonal touch: Expressing and recognizing emotions through haptic devices," Human-Computer Interaction, vol. 22, no. 3, pp. 325-353, 2007.

[8] A. Haans and W. IJsselsteijn, "Mediated social touch: a review of current research and future directions," Virtual Reality, vol. 9, no. 2-3, pp. 149-159, 2006.

[9] G. Huisman, A. Darriba Frederiks, E. M. A. G. Van Dijk, D. K. J Heylen, and B. J. A. Kröse, "The TaSST - Tactile Sleeve for Social Touch," in Proceedings of WHC'13. IEEE, 2013, pp. 211-216.

[10] I. Oakley, Y. Kim, J. Lee, and J. Ryu, "Determining the Feasibility of Forearm Mounted Vibrotactile Displays," in Proceedings of HAP TICS'06. IEEE, 2006, pp. 27 - 34

[11] G. Huisman and A. Darriba Frederiks, "Towards Tactile Expressions of Emotion Through Mediated Touch," in Proceedings of CHI'13. ACM, 2013, pp. 1575-1580. 